Check for updates

Institute of Psychiatry, Psychology and Neuroscience, King's College London, London, UK

2 Centre for Clinical Brain Sciences, University of Edinburgh, Edinburgh, UK

3 Department of Neurology, Walton Centre NHS Foundation Trust, Liverpool, UK

Correspondence to: T R Nicholson timothy.nicholson@kcl.ac.uk Cite this as: BMJ 2020;371:m3871 http://dx.doi.org/10.1136/bmj.m3871 Published: 13 October 2020

\title{
Neuropsychiatric complications of covid-19
}

\author{
From acute delirium to long term fatigue, covid-19 has serious neuropsychiatric effects \\ Matthew Butler, ${ }^{1}$ Thomas A Pollak, ${ }^{1}$ Alasdair G Rooney, ${ }^{2}$ Benedict D Michael, ${ }^{3}$ Timothy R Nicholson ${ }^{1}$
}

Viral infections of the respiratory tract can have multisystemic effects, including on the central nervous system (CNS), and thus may precipitate a spectrum of psychiatric and neurological disorders. ${ }^{1}$ Some patients with covid-19 are now known to develop various CNS abnormalities with potentially serious and long term consequences, including stroke and isolated psychiatric syndromes. ${ }^{2}$ As covid-19 cases rise again worldwide, it is timely to review what we know and don't know about the acute and chronic neuropsychiatric sequelae and their potential mechanisms.

In laboratory studies, SARS-CoV-2 is neurotropic (replicating in neurons) and has the potential to enter the CNS via angiotensin converting enzyme 2 receptors in the olfactory bulb. ${ }^{3}$ Whether this happens in clinical populations is unclear. Postmortem studies show evidence of cerebral inflammation, and neuroimaging studies detect leucoencephalopathy and microbleeds in critically ill patients. ${ }^{4}$ While viral RNA is typically absent from the cerebrospinal fluid 5 and postmortem brain samples, ${ }^{6}$ antibodies to SARS-CoV-2 have been identified in cerebrospinal fluid. ${ }^{7}$ Other mechanisms causing CNS dysfunction include cytokine driven inflammatory responses ${ }^{8}$ and wider systemic effects such as endotheliopathy, breakdown of the blood-brain barrier, ${ }^{7}$ and clotting abnormalities. ${ }^{6}$

Additional biological mechanisms, including autoimmunity, may be relevant to psychiatric disorders, as well as the psychological trauma of life threatening illness and pandemic related socioeconomic stressors. The relative contributions of these mechanisms to neuropsychiatric complications remain largely unknown.

\section{Acute neuropsychiatric symptoms}

Neuropsychiatric symptoms, particularly delirium, were common in previous coronavirus outbreaks of severe acute respiratory disease (SARS) and Middle East respiratory syndrome (MERS). ${ }^{9}$ So far, covid-19 seems to follow a similar pattern: delirium is the most common acute neuropsychiatric syndrome ${ }^{10}$ and may be the sole presenting feature of covid-19 in older adults and those with dementia. ${ }^{11}$ Delirium is associated with poorer outcomes ${ }^{10}$ and is especially prevalent among patients requiring intensive care, ${ }^{12}$ where cognitive and behavioural abnormalities have been reported in one third of patients after discharge. ${ }^{13}$

Screening for delirium should be considered in acute cases, particularly in older adults or those with pre-existing dementia, using a validated tool such as the $4 \mathrm{AT}$ (the 4 at.com ${ }^{14}$, along with close monitoring for longer term adverse outcomes such as cognitive impairment. ${ }^{15}$ Efforts should also be made to identify patients with alterations in mental state that are not explained by delirium, as they may need more advanced diagnostic and therapeutic approaches.

During previous coronavirus pandemics, low mood and anxiety were common in the acute phase of infection (with psychosis and catatonia in a minority), and in one study of covid-19 patients admitted with acute respiratory distress syndrome, fatigue persisted after discharge in around one fifth. ${ }^{9}$ The UK based CoroNerve group is gathering surveillance data through clinician reports of neurological and neuropsychiatric disorders among inpatients with covid-19. Reports so far include covid-19 associated psychosis, mood disorders, and catatonia as well as encephalopathy, encephalitis, and other neurological disorders in those admitted with covid-19. ${ }^{16}$ We encourage clinicians encountering such cases to report them through the study website (coronerve.com). ${ }^{17}$ The precise incidence of these conditions and whether they are also associated with milder forms of covid-19 are still unclear.

High rates of anxiety, depression, and post-traumatic stress disorder have been reported in people who recover after hospital admission for covid-19, ${ }^{18}$ but it is still too early to gauge the full effect of long term complications. A complete picture may not emerge for years, as was the case when children born during influenza pandemics were found to have higher rates of psychotic disorders in adulthood. ${ }^{1}$

\section{Long covid}

However, some initial observations can be made. Some patients treated in hospital for severe covid-19 experience disabling fatigue ${ }^{19}$ and cognitive difficulties ${ }^{20}$ after discharge. A diverse group of presentations, increasingly referred to as "long covid," is also being described in patients who did not require hospital treatment. These patients report a wide variety of symptoms, persisting for many months after acute infection. To date, most research on long covid has been led by patients, and it suggests this is a common and disabling disorder..$^{21}$ Further research informing clinical guidelines and our understanding of the mechanisms underlying this disorder is urgently required. ${ }^{22}$

Covid-19 is likely to have important neuropsychiatric effects in both the short and longer term. Emerging data suggest delirium is the most common acute neuropsychiatric manifestation, and both screening at-risk individuals and long term monitoring should be considered. Acute psychiatric complications are also a concern, and epidemiological and mechanistic studies are needed to augment current surveillance efforts. 
Looking to the future, long covid is likely to result in substantial population morbidity with serious implications for health and social care. Urgent research is needed to characterise this disorder, define disease mechanisms, direct the use of either existing therapeutics or the development of novel treatments, and direct health and social care support. Clinical scientists must work closely with patient groups and national and international organisations such as the WHO Brain Health Unit. Collaboration will help to translate research findings into meaningful improvements in managing the acute and long term neuropsychiatry of covid-19. We strongly support public health messaging emphasising longer term morbidity, including neuropsychiatric complications, associated with covid-19.

Competing interests: The BM/ has judged that there are no disqualifying financial ties to commercial companies. The authors declare no other interests. The BMJ policy on financial interests is here: https://www.bmj.com/sites/default/files/attachments/resources/2016/03/16-current-bmj-educationcoi-form.pdf

Provenance and peer review: Commissioned; not externally peer reviewed.

1 Kępińska AP, Iyegbe CO, Vernon AC, Yolken R, Murray RM, Pollak TA. Schizophrenia and influenza at the centenary of the 1918-1919 Spanish influenza pandemic: mechanisms of psychosis risk. Front Psychiatry 2020;11:72. doi: 10.3389/fpsyt.2020.00072 pmid: 32174851

2 Butler M, Watson C, Rooney A, et al. The neurology and neuropsychiatry of covid-19. BMJ Opinion, 1 May 2020. https://blogs.bmj.com/jnnp/2020/05/01/the-neurology-and-neuropsychiatry-ofcovid-19/

3 ladecola C, Anrather J, Kamel H. Effects of covid-19 on the nervous system. Cell2020;183:16-27.e1. doi: 10.1016/j.cell.2020.08.028 pmid: 32882182

4 Gulko E, Oleksk ML, Gomes W, etal. MRI brain findings in 126 patients with covid-19: initial observations from a descriptive literature review. AJNR Am J Neuroradiol 2020. doi: 10.3174/ajnr.A6805 pmid: 32883670

5 Neumann B, Schmidbauer ML, Dimitriadis K, etalPANDEMIC and the IGNITE study groups. Cerebrospinal fluid findings in COVID-19 patients with neurological symptoms. J Neurol SCi 2020;418:117090. doi: 10.1016/j.jns.2020.117090 pmid: 32805440

6 Al-Sarraj S, Troakes C, Hanley B, etal. The spectrum of neuropathology in COVID-19. Neuropathol Appl Neurobiol 2020 [Epub ahead of print]. doi: 10.1111/nan.12667 pmid: 32935873

7 Alexopoulos H, Magira E, Bitzogli K, etal. Anti-SARS-CoV-2 antibodies in the CSF, blood-brain barrier dysfunction, and neurological outcome: Studies in 8 stuporous and comatose patients. Neurol Neuroimmunol Neuroinflamm 2020;7:e893. doi: 10.1212/NXI.0000000000000893 pmid: 32978291

8 Mehta P, McAuley DF, Brown M, Sanchez E, Tattersall RS, Manson JJHLH Across Speciality Collaboration, UK. COVID-19: consider cytokine storm syndromes and immunosuppression. Lancet 2020;395:1033-4. doi: 10.1016/S0140-6736(20)30628-0 pmid: 32192578

9 Rogers JP, Chesney E, Oliver D, etal. Psychiatric and neuropsychiatric presentations associated with severe coronavirus infections: a systematic review and meta-analysis with comparison to the COVID-19 pandemic. Lancet Psychiatry 2020;7:611-27. doi: 10.1016/S2215-0366(20)30203-0 pmid: 32437679

10 Mcloughlin BC, Miles A, Webb TE, etal. Functional and cognitive outcomes after COVID-19 delirium. Eur Geriatr Med 2020. doi: 10.1007/s41999-020-00353-8 pmid: 32666303

11 Poloni TE, Carlos AF, Cairati M, etal. Prevalence and prognostic value of delirium as the initial presentation of COVID-19 in the elderly with dementia: an Italian retrospective study. EClinicalMedicine 2020;26:100490. doi: 10.1016/j.eclinm.2020.100490 pmid: 32838241

12 Helms J, Kremer S, Merdji H, etal. Delirium and encephalopathy in severe COVID-19: a cohort analysis of ICU patients. Crit Care 2020;24:491. doi: 10.1186/s13054-020-03200-1 pmid: 32771053

13 Helms J, Kremer S, Merdji H, etal. Neurologic features in severe SARS-CoV-2 infection. N Engl J Med 2020doi: 10.1056/NEJMc2008597.

14 Shenkin SD, Fox C, Godfrey M, etal. Delirium detection in older acute medical inpatients: a multicentre prospective comparative diagnostic test accuracy study of the 4AT and the confusion assessment method. BMC Med 2019;17:138. doi: 10.1186/s12916-019-1367-9 pmid: 31337404

15 Scottish Intercollegiate Guidelines Network. Sign 157: risk reduction and management of delirium: a national clinical guideline. SIGN, 2019. https://www.sign.ac.uk/sign-157-delirium

16 Varatharaj A, Thomas N, Ellul MA, etalCoroNerve Study Group. Neurological and neuropsychiatric complications of COVID-19 in 153 patients: a UK-wide surveillance study. Lancet Psychiatry 2020;7:875-82. doi: 10.1016/S2215-0366(20)30287-X pmid: 32593341

17 Royal College of Psychiatrists. Coronerve surveillance survey. https://www.rcpsych.ac.uk/members/your-faculties/neuropsychiatry/coronerve-surveillance-survey

18 Mazza MG, De Lorenzo R, Conte C, etalCOVID-19 BioB Outpatient Clinic Study group. Anxiety and depression in COVID-19 survivors: Role of inflammatory and clinical predictors. Brain Behav Immun 2020;S0889-1591(20)31606-8. doi: 10.1016/j.bbi.2020.07.037 pmid: 32738287

19 Halpin SJ, Mclvor C, Whyatt G, etal. Postdischarge symptoms and rehabilitation needs in survivors of COVID-19 infection: A cross-sectional evaluation. J Med Virol 2020.

doi: 10.1002/jmv.26368 pmid: 32729939
20 Zhou H, Lu S, Chen J, etal. The landscape of cognitive function in recovered COVID-19 patients. J Psychiatr Res 2020;129:98-102. doi: 10.1016/j.jpsychires.2020.06.022 pmid: 32912598

21 Body Politic COVID-19 Support Group. Report: what does covid-19 recovery actually look like? 2020. https://patientresearchcovid19.com/research/report-1/

22 Greenhalgh T, Knight M, A'Court C, Buxton M, Husain L. Management of post-acute covid-19 in primary care. BM/2020;370:m3026. doi: 10.1136/bmj.m3026 pmid: 32784198

This article is made freely available for use in accordance with BMJ's website terms and conditions for the duration of the covid-19 pandemic or until otherwise determined by BMJ. You may use, download and print the article for any lawful, non-commercial purpose (including text and data mining) provided that all copyright notices and trade marks are retained. 